\title{
Oral self-administration of ethanol, phencyclidine, methadone, pentobarbital and quinine in rhesus monkeys
}

\begin{abstract}
Rationale: Simultaneous and sequential drug use among clinical populations is the norm, whereas the pattern of self-administration of multiple drugs among non-human primate populations has not been thoroughly explored. Objectives: To determine the relationship between the preferences and intakes of a large group of rhesus monkeys exposed to various orally available solutions. Methods: Thirteen male and eleven female young adult rhesus monkeys (Macaca mulatta) were exposed to orally available drug solutions using a concurrent choice (drug and water) procedure, where fluid delivery was made contingent upon single spout contacts (fixed ratio one). Results: Ethanol $(0.25-16 \% \mathrm{w}: \mathrm{v})$ produced biphasic effects on the number of fluid deliveries obtained, with peak ethanol preferences over water demonstrated at the 1-2\% w:v concentrations. No preferences for the $N$-methyl-D-aspartate receptor antagonist phencyclidine or water were demonstrated at lower concentrations $(0.0078125-0.125 \mathrm{mg} / \mathrm{ml})$ and, at higher concentrations $(0.25,0.5 \mathrm{mg} / \mathrm{ml})$, a preference for water was demonstrated. The $\mu$ opioid receptor agonist methadone $(0.001-0.3 \mathrm{mg} / \mathrm{ml})$ and the prototypic bitter substance quinine $(0.001-0.3 \mathrm{mg} / \mathrm{ml})$ failed to produce
\end{abstract}

M. Linnoila deceased

J.A. Vivian $(\varangle) \cdot$ Y.J. Liang · J.H. Woods

Department of Pharmacology,

University of Michigan Medical School, 1301, MSRB III,

Ann Arbor, MI 48109-0632, USA

e-mail: jvivian@umich.edu, Tel.: +1-734-6473119,

Fax: +1-734-7647118

J.H. Woods

Department of Psychology, University of Michigan,

Ann Arbor, Michigan, USA

J.D. Higley $\cdot$ M. Linnoila

Laboratory of Clinical Studies - Primate Unit,

DICBR, NIAAA, NIH, Poolesville, Maryland, USA

Present address:

J.A. Vivian, Department of Physiology and Pharmacology,

Wake Forest University School of Medicine,

Medical Center Boulevard, Winston-Salem, NC 27157, USA

Tel.: +1-336-7168552, Fax: +1-336-7168501 preferences for drug or water. A large preference for water over the barbiturate pentobarbital $(0.01-3 \mathrm{mg} / \mathrm{ml})$ was also demonstrated. After rank-ordering the subjects based on their drug preferences or intakes, modest to no correlations across drugs were demonstrated. Conclusions: These results reveal that a robust ethanol preference is not predictive of a preference for drugs of abuse from other classes and suggests that fluid intakes were correlated, irrespective of the presence or absence of drug in the solution.

Key words Barbiturates $\cdot$ Ethanol $\cdot$ Individual differences - NMDA - Opiates · Preferences · Quinine · Self-administration

\section{Introduction}

Many risk factors have been proposed that may account for an organism's propensity to use or abuse drugs. Genetic factors involved in alcohol abuse have been consistently implicated through the use of family, twin and adoption studies (Merikangas et al. 1993). Various psychopathologies, including impulse-control problems, have proven predictive of substance abuse (Tarter and Mezzich 1993). In addition, environmental factors, such as drug exposure and availability, and stressful early life and poor social experiences are associated with augmented drug use (Cadoret 1993). It has been further suggested that specific drugs (i.e., nicotine, alcohol, marijuana) may serve as "gateways" to future, and other, drug use (Torabi et al. 1993). In a similar vein, for those individuals who use a drug, the exclusive use of one drug throughout the lifetime is unusual (Newcomb 1993). In fact, comorbid drug use is common in clinical drug-using populations (Martin et al. 1996) and has led to the proposal that drug-seeking behavior may have common biological determinants (George 1993).

Although multiple-drug use is the norm in human populations, preclinical research detailing individual patterns of preferences and consumption across orally avail- 
Fig. 1 Approximate behavioral histories of the NIAAA and MICH monkeys serving as subjects for the current experiment

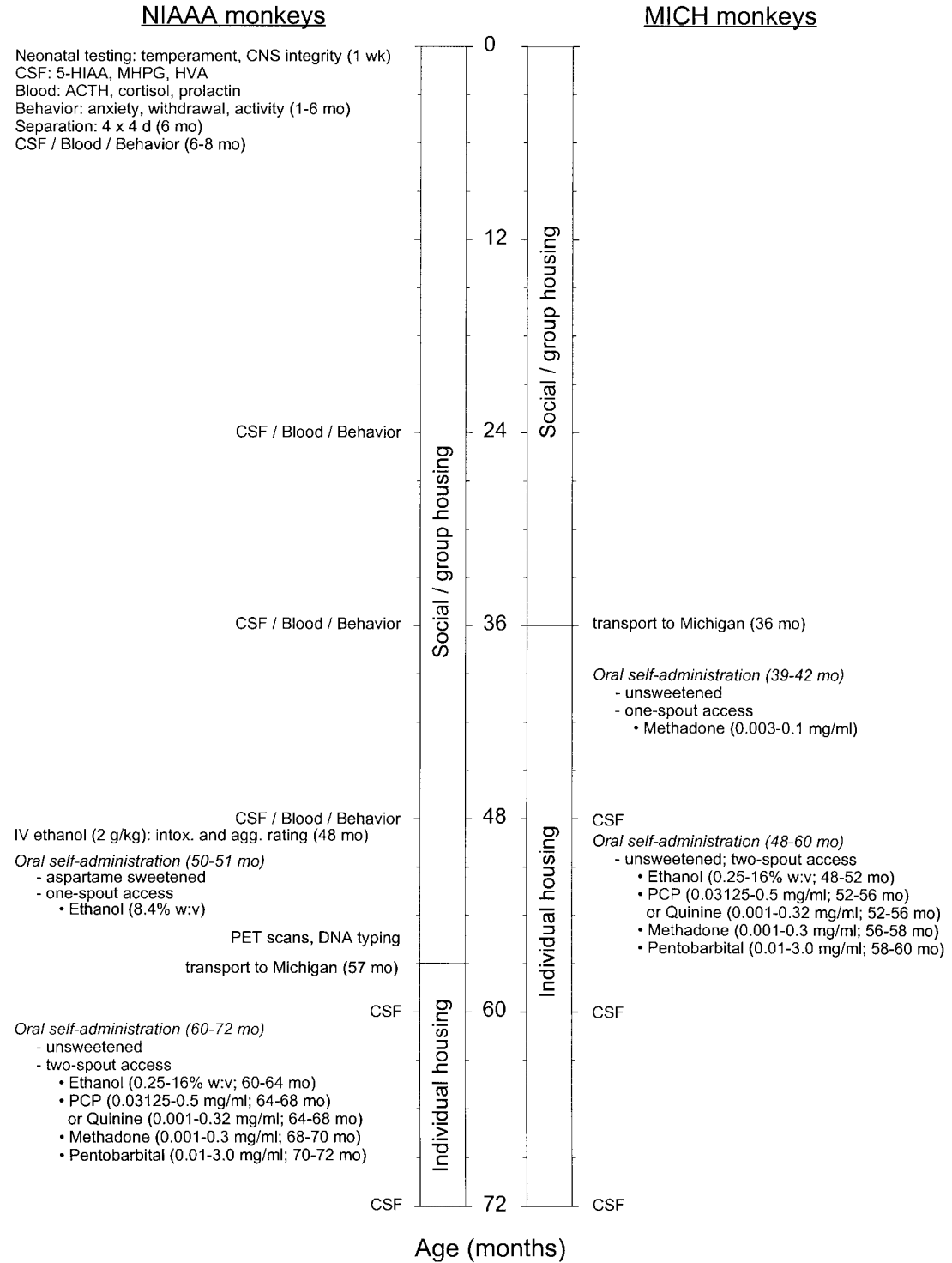

able drugs has been surprisingly sparse. In rats, correlations have been demonstrated between cocaine and ethanol (Gahtan et al. 1996), yet no such association was demonstrated among etonitazene, cocaine and ethanol (Carlson and Perez 1997). In rhesus monkeys, self-administration of ethanol was not correlated with self-administration of cocaine or midazolam (Meisch et al. 1990; Stewart et al. 1994). In the current investigation, a large group of rhesus monkeys $(n=24)$ were exposed to ethanol and drugs from various pharmacological classes: the $N$-methyl-D-aspartate (NMDA) antagonist phencyclidine, the $\mu$ opiate methadone and the barbiturate pentobarbital. Monkeys were also exposed to the prototypic bitter substance quinine. These compounds were chosen due to their well-documented oral-reinforcing properties (ethanol: Henningfield and Meisch 1978; phencyclidine: Carroll 1982; methadone: Stewart et al. 1996; pentobarbital: Meisch et al. 1981) or salient taste characteristics without notable reinforcing properties (quinine: Aspen et al. 1999; Vivian et al. 1999b) in an effort to evaluate the relationship between preferences and intakes among multiple orally available solutions.

\section{Methods}

Subjects

Thirteen adult male and eleven female young-adult rhesus monkeys (Macaca mulatta) were individually housed with free access to water in a vivarium maintained at $21 \pm 1^{\circ} \mathrm{C}, 30-50 \%$ humidity and a 12-h/12-h light/dark cycle. Monkeys were allowed to maintain or increase their weight and were fed 20-30 biscuits (Purina Monkey Chow) daily and fresh fruit twice weekly. Experimental sessions were performed in the home cage.

The experimental history of the monkeys have been previously described (Vivian et al. 1999a). Briefly, all monkeys were grouphoused in large indoor-outdoor pens through age 36 months and were allowed to interact freely and establish social hierarchies with free access to food and water. Prior to the current experiment, one-half of the monkeys (NIAAA; $n=12$ ) were given a single administration of ethanol ( $\mathrm{g} / \mathrm{kg}$ i.v. $)$, and single-spout oral ethanol self-administration experiments were performed (Higley et al. 
1991, 1996). The remaining twelve monkeys (MICH) were exposed to unsweetened methadone using a single-spout oral selfadministration procedure. For the current experiment, all monkeys were exposed to single-spout panel training sessions, followed by two-spout oral self-administration testing sessions (described below). The approximate experimental histories of the monkeys are depicted in Fig. 1.

\section{Apparatus}

An operant panel for fluid delivery and response measurement was attached to one wall of the home cage $(61 \times 69 \times 76 \mathrm{~cm})$. This panel was composed of two drinking spouts (D: $1.2 \mathrm{~cm}, \mathrm{~L}: 2.7 \mathrm{~cm}$ ) protruding into the cage, positioned $30 \mathrm{~cm}$ from each other, below two light-emitting diodes (LEDs). Lip contacts to the drinking spouts defined a response and completed a drinkometer circuit which opened a solenoid for fluid delivery $(0.5 \mathrm{ml})$. The LEDs were illuminated green during the session to signal fluid availability, and red to signal spout contact. Additional discriminative stimuli associated with spout contact were provided by an audible click when the solenoids were operated. Experimental sessions were controlled and data were collected in an adjacent room with IBM-compatible computers.

Procedure

\section{Self-administration training}

Monkeys were initially exposed to daily 2-h sessions comprised of water access through one of the two spouts. Delivery of the fluid $(0.5 \mathrm{ml})$ was contingent upon one spout contact (fixed ratio one; FR 1). The side that delivered water was determined randomly in blocks of six, and the daily food regimen was delivered immediately prior to the session to promote drinking behavior (e.g., prandial drinking). NIAAA and MICH monkeys were exposed to this training condition for 11 and 30 sessions, respectively (when intrasession water self-administration varied by less than 10\%); drug solutions were not available during this portion of the experiment.

\section{Self-administration testing}

Monkeys were exposed to daily 2-h sessions comprised of concurrent access to individual drug solutions and water through both spouts. Fluid delivery $(0.5 \mathrm{ml})$ was contingent upon one spout contact (FR 1). The side that delivered the drug solution or water was determined randomly in blocks of six, and the daily food regimen was delivered after the experimental session (i.e., there were no induction procedures). Each drug concentration was made available for six sessions before advancement to the next concentration [the duration of ethanol exposure (6-day through 30-day determinations of a single concentration) has been found not to influence the preferences or consumption of ethanol in this collection of monkeys].

Drugs

Ascending concentrations of unsweetened ethanol (0.25-16\% w:v; McCormick Distillery, Weston, Mo.), phencyclidine (0.0078125$0.5 \mathrm{mg} / \mathrm{ml}$; National Institute on Drug Abuse, Rockville, Md.), methadone $(0.001-0.3 \mathrm{mg} / \mathrm{ml}$; National Institute on Drug Abuse), pentobarbital (0.01-3 mg/ml; National Institute on Drug Abuse) or quinine $(0.001-0.3 \mathrm{mg} / \mathrm{ml}$; Sigma Chemical, St. Louis, Mo.) were made available in blocks of six sessions, with replications of selected concentrations performed in a varied order (i.e., ethanol: 0 , $2,0.25,8,1 \% \mathrm{w} / \mathrm{v})$. The order of drug presentation was ethanol, phencyclidine or quinine, methadone and pentobarbital. For onehalf of the subjects, exposure to phencyclidine was performed prior to quinine determinations, for the other one-half of the subjects, exposure to quinine was performed prior to phencyclidine determinations.

Data analysis

Mean drug and water fluid deliveries and drug intake $(\mathrm{mg} / \mathrm{kg})$ were calculated for each drug concentration (data from six sessions). Mean drug and water fluid deliveries were analyzed using a four-factor (group, gender, drug, concentration) within-subjects analysis of variance (ANOVA). Mean drug intake was analyzed using a three-factor (group, gender, concentration) within-subjects ANOVA. When significant effects were demonstrated, post-hoc comparisons utilizing the $95 \%$ confidence intervals associated with a mean were performed with non-overlapping confidence intervals accepted as significant.

An alternative analysis of drug self-administration was established by comparing the area under the curve (AUC) that described drug and water fluid deliveries, and the AUC that described drug intake, across the tested drug concentrations. Briefly, AUC estimates were calculated for each monkey by integrating mean drug and water fluid deliveries or mean drug intake $\left(\mathrm{AUC}_{\text {DrugFD }}, \mathrm{AUC}_{\text {WaterFD }}, \mathrm{AUC}_{\mathrm{INT}}\right.$, respectively) across drug concentration using a statistical package for pharmacology (Tallarida and Murray 1986). Drug preference was defined as the difference between drug and water fluid delivery curves $\left(\triangle \mathrm{AUC} \mathrm{C}_{\mathrm{FD}}\right)$. Test-retest reliability measures exceeded $r=0.8$ for all AUC measures. In addition, the drug concentration that produced the largest mean drug preferences (drug/water fluid deliveries) or aversions (water/drug fluid deliveries) for each drug was determined. After rank-ordering subjects based on preferences or intakes, Spearman correlation coefficients were calculated in the analysis of the relationship between preferences or intakes between drugs. Finally, because baseline intakes of water in water-water choice conditions were correlated with drug intakes, partial correlations between individual drugs were performed, controlling for baseline water intake (for detail, see Kachigan 1986). For all comparisons, alpha was 0.05 , two-tailed.

\section{Results}

In general and for all of the drug solutions and concentrations tested, there were notable differences between NIAAA and MICH monkeys. NIAAA monkeys obtained more baseline and drug fluid deliveries than MICH monkeys. Similarly, NIAAA monkeys consumed $(\mathrm{mg} / \mathrm{kg})$ more drug than MICH monkeys. Although NIAAA monkeys were polydipsic compared with MICH monkeys, monkeys in each group responded to changes in drug concentration in a similar and orderly fashion.

\section{Ethanol}

\section{Fluid deliveries}

Ethanol produced biphasic effects on the number of fluid deliveries obtained, with peak ethanol preferences demonstrated at the 1-2\% w:v concentrations (Fig. 2, top panels). Ethanol (0.25-4\% w:v) fluid deliveries were greater than baseline water-water choice trials (i.e., ethanol $0 \% \mathrm{w}: \mathrm{v})$ and concurrent water fluid deliveries; water fluid deliveries were greater than baseline and ethanol fluid deliveries when tested concurrently with ethanol (16\% w:v). There were no gender differences (main ef- 
Fig. 2 Top panels: ethanol (filled symbols) and water (hollow symbols) fluid deliveries (per session) as a function of ethanol concentration (\% w:v). Unconnected symbols indicate the fluid deliveries obtained in sessions in which water was concurrently available from both spouts. Bottom panels: ethanol intake (mg/kg per session) as a function of ethanol concentration (\% w:v). Left and right panels depict NIAAA and MICH monkeys, respectively. For all panels, each point represents the mean of six sessions, and error bars denote the 95\% confidence interval associated with the mean
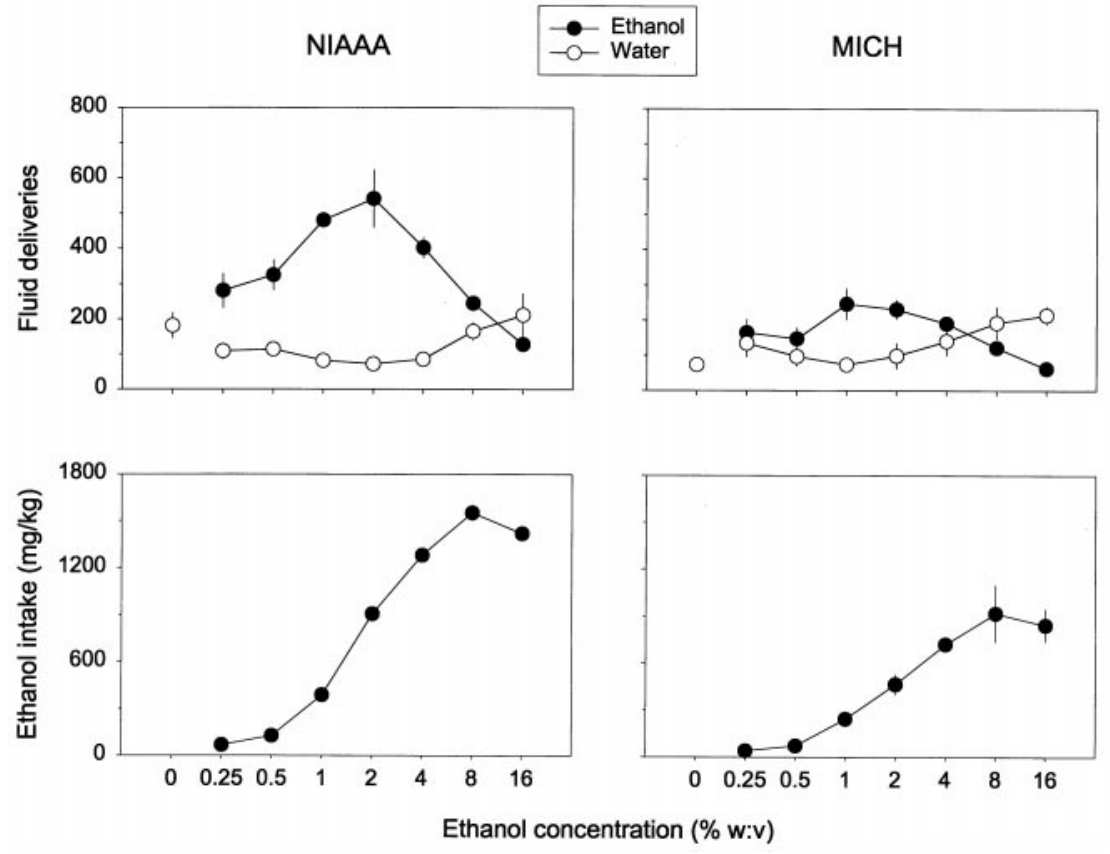

Table 1 Oral ethanol self-administration in rhesus monkeys

* Significant $F$ value $(P<0.05)$ for comparison

\begin{tabular}{llll}
\hline Factor & df & $F$ value & $P$ value \\
\hline Fluid deliveries & & & \\
Group & 1,20 & 2.20 & 0.153 \\
Gender & 1,20 & 0.36 & 0.553 \\
Drug & 1,20 & 16.37 & $0.001^{*}$ \\
Concentration & 7,140 & 5.48 & $0.001^{*}$ \\
Group $\times$ Gender & 1,20 & 0.60 & 0.448 \\
Group $\times$ Drug & 1,20 & 9.38 & $0.006^{*}$ \\
Group $\times$ Concentration & 7,140 & 1.79 & 0.093 \\
Gender $\times$ Drug & 1,20 & 1.47 & 0.240 \\
Gender $\times$ Concentration & 7,140 & 1.95 & 0.066 \\
Drug $\times$ Concentration & 7,140 & 14.65 & $0.001^{*}$ \\
Group $\times$ Gender $\times$ Drug & 1,20 & 1.71 & 0.292 \\
Group $\times$ Gender $\times$ Concentration & 7,140 & 1.59 & 0.144 \\
Group $\times$ Drug $\times$ Concentration & 7,140 & 1.85 & 0.082 \\
Gender $\times$ Drug $\times$ Concentration & 7,140 & 0.59 & 0.762 \\
Group $\times$ Gender $\times$ Drug $\times$ Concentration & 7,140 & 0.41 & 0.894 \\
Intake & & & \\
Group & & & \\
Gender & 1,20 & 8.48 & $0.009^{*}$ \\
Concentration & 1,20 & 0.03 & 0.866 \\
Group $\times$ Gender & 7,140 & 80.83 & $0.001^{*}$ \\
Group $\times$ Concentration & 1,20 & 0.02 & 0.962 \\
Gender $\times$ Concentration & 7,140 & 5.70 & $0.001^{*}$ \\
Group $\times$ Gender $\times$ Concentration & 7,140 & 0.12 & 0.997 \\
\hline & 7,140 & 0.73 & 0.646 \\
\hline
\end{tabular}

fects and interactions) in ethanol or water fluid deliveries (Table 1).

\section{Intake}

Self-administered ethanol intake $(\mathrm{mg} / \mathrm{kg})$ increased as a function of ethanol concentration $(0.25-8 \% \mathrm{w}: \mathrm{v})$, reaching significance at the $1 \% \mathrm{w}: \mathrm{v}$ concentration (Fig. 2, bottom panels). Maximal ethanol intake was demonstrated at the $8 \%(\mathrm{w}: \mathrm{v})$ concentration, in which NIAAA and
MICH monkeys consumed approximately $1600 \mathrm{mg} / \mathrm{kg}$ and $800 \mathrm{mg} / \mathrm{kg}$, respectively. As with fluid deliveries, there were no gender differences in ethanol intake.

Phencyclidine

\section{Fluid deliveries}

Phencyclidine $(0.0078125-0.125 \mathrm{mg} / \mathrm{ml})$ produced inconsistent effects on the number of fluid deliveries ob- 
Fig. 3 Top panels: phencyclidine (filled symbols) and water (hollow symbols) fluid deliveries (per session) as a function of phencyclidine concentration $(\mathrm{mg} / \mathrm{ml})$. Unconnected symbols indicate the fluid deliveries obtained in sessions in which water was concurrently available from both spouts. Bottom panels: phencyclidine intake (mg/kg per session) as a function of phencyclidine concentration $(\mathrm{mg} / \mathrm{ml})$. Left and right panels depict NIAAA and MICH monkeys, respectively. For all panels, each point represents the mean of six sessions, and error bars denote the 95\% confidence interval associated with the mean

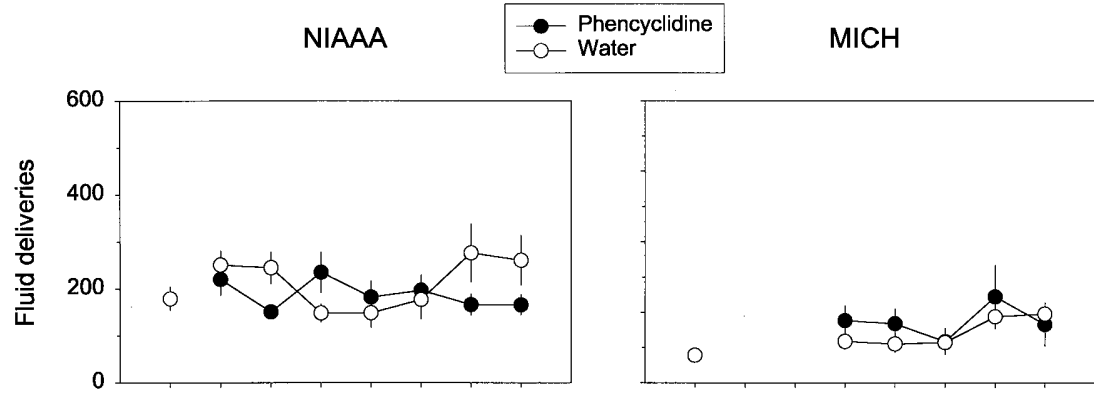

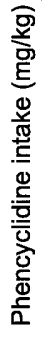
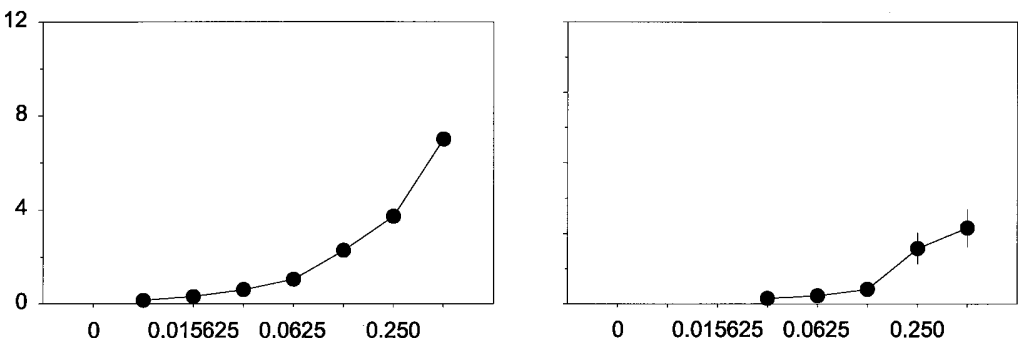

Phencyclidine concentration ( $\mathrm{mg} / \mathrm{ml})$
Table 2 Oral phencyclidine self-administration in rhesus monkeys
* Significant $F$ value $(P<0.05)$ for comparison

\begin{tabular}{llll}
\hline Factor & df & $F$ value & $P$ value \\
\hline Fluid deliveries & & & \\
Group & 1,20 & 11.44 & $0.003^{*}$ \\
Gender & 1,20 & 0.001 & 0.981 \\
Drug & 1,20 & 0.004 & 0.949 \\
Concentration & 5,100 & 5.61 & $0.001^{*}$ \\
Group $\times$ Gender & 1,20 & 0.13 & 0.725 \\
Group $\times$ Drug & 1,20 & 0.61 & 0.443 \\
Group $\times$ Concentration & 5,100 & 0.60 & 0.699 \\
Gender $\times$ Drug & 1,20 & 0.27 & 0.609 \\
Gender $\times$ Concentration & 5,100 & 0.65 & 0.665 \\
Drug $\times$ Concentration & 5,100 & 3.50 & $0.006^{*}$ \\
Group $\times$ Gender $\times$ Drug & 1,20 & 2.20 & 0.153 \\
Group $\times$ Gender $\times$ Concentration & 5,100 & 0.59 & 0.709 \\
Group $\times$ Drug $\times$ Concentration & 5,100 & 2.50 & $0.035^{*}$ \\
Gender $\times$ Drug $\times$ Concentration & 5,100 & 0.48 & 0.789 \\
Group $\times$ Gender $\times$ Drug $\times$ Concentration & 5,100 & 0.97 & 0.438 \\
Intake & & & \\
Group & & & \\
Gender & 1,20 & 22.37 & $0.001^{*}$ \\
Concentration & 1,20 & 2.67 & 0.118 \\
Group $\times$ Gender & 7,140 & 44.04 & $0.001^{*}$ \\
Group $\times$ Concentration & 1,20 & 1.56 & 0.226 \\
Gender $\times$ Concentration & 7,140 & 12.13 & $0.001^{*}$ \\
Group $\times$ Gender $\times$ Concentration & 7,140 & 1.11 & 0.362 \\
\hline & 7,140 & 0.77 & 0.576 \\
\hline
\end{tabular}

tained in NIAAA and MICH monkeys (i.e., no preferences for either fluid were demonstrated). At higher phencyclidine concentrations $(0.25,0.5 \mathrm{mg} / \mathrm{ml})$, more water than phencyclidine fluid deliveries were obtained by the NIAAA monkeys (Fig. 3, top panels). No gender differences (main effects and interactions) were demonstrated with phencyclidine or water fluid deliveries (Table 2).

\section{Intake}

Self-administered phencyclidine $(\mathrm{mg} / \mathrm{kg})$ intake increased as a function of phencyclidine concentration, reaching significance at the $0.125 \mathrm{mg} / \mathrm{ml}$ concentration (Fig. 3, bottom panels). Maximal phencyclidine intake was demonstrated at the highest tested concentration $(0.5 \mathrm{mg} / \mathrm{ml})$, in which NIAAA and MICH monkeys consumed approximately $7 \mathrm{mg} / \mathrm{kg}$ and $2 \mathrm{mg} / \mathrm{kg}$, respectively. There were no gender differences in phencyclidine intake. 
Fig. 4 Top panels: methadone (filled symbols) and water (hollow symbols) fluid deliveries (per session) as a function of methadone concentration (mg/ml). Unconnected symbols indicate the fluid deliveries obtained in sessions in which water was concurrently available from both spouts. Bottom panels: methadone intake $(\mathrm{mg} / \mathrm{kg}$ per session) as a function of methadone concentration $(\mathrm{mg} / \mathrm{ml})$. Left and right panels depict NIAAA and MICH monkeys, respectively. For all panels, each point represents the mean of six sessions, and error bars denote the $95 \%$ confidence interval associated with the mean

Table 3 Oral methadone selfadministration in rhesus monkeys

* Significant $F$ value $(P<0.05)$ for comparison
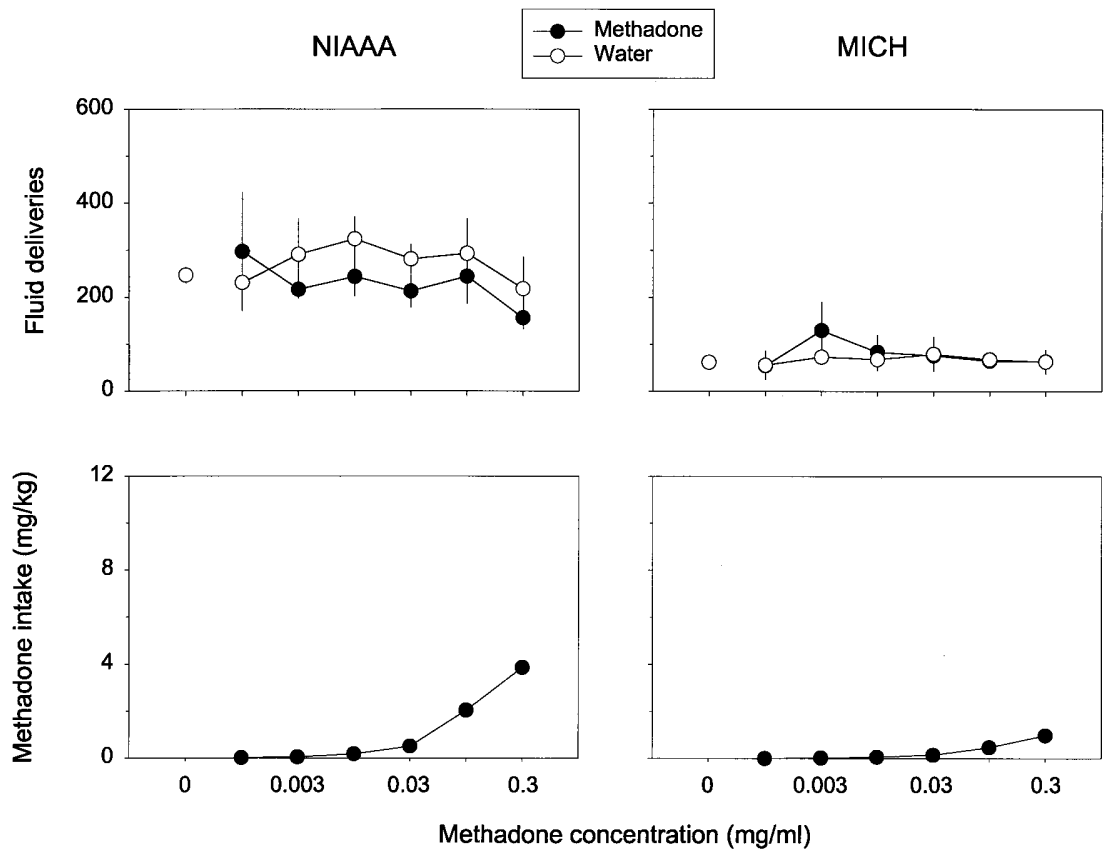

\begin{tabular}{llll}
\hline Factor & df & $F$ value & $P$ value \\
\hline Fluid deliveries & & & \\
Group & 1,20 & 3.93 & 0.061 \\
Gender & 1,20 & 1.00 & 0.328 \\
Drug & 1,20 & 0.31 & 0.586 \\
Concentration & 6,120 & 2.22 & $0.046^{*}$ \\
Group $\times$ Gender & 1,20 & 1.36 & 0.257 \\
Group $\times$ Drug & 1,20 & 0.99 & 0.333 \\
Group $\times$ Concentration & 6,120 & 1.52 & 0.178 \\
Gender $\times$ Drug & 1,20 & 0.41 & 0.527 \\
Gender $\times$ Concentration & 6,120 & 1.67 & 0.133 \\
Drug $\times$ Concentration & 6,120 & 0.37 & 0.897 \\
Group $\times$ Gender $\times$ Drug & 1,20 & 0.40 & 0.534 \\
Group $\times$ Gender $\times$ Concentration & 6,120 & 2.03 & 0.067 \\
Group $\times$ Drug $\times$ Concentration & 6,120 & 0.78 & 0.584 \\
Gender $\times$ Drug $\times$ Concentration & 6,120 & 0.66 & 0.682 \\
Group $\times$ Gender $\times$ Drug $\times$ Concentration & 6,120 & 0.25 & 0.958 \\
& & & \\
Intake & & & \\
Group & 1,20 & 4.13 & 0.056 \\
Gender & 1,20 & 0.77 & 0.390 \\
Concentration & 6,120 & 10.23 & $0.001 *$ \\
Group $\times$ Gender & 1,20 & 1.36 & 0.258 \\
Group $\times$ Concentration & 6,120 & 3.66 & $0.002^{*}$ \\
Gender $\times$ Concentration & 6,120 & 0.94 & 0.467 \\
Group $\times$ Gender $\times$ Concentration & 6,120 & 1.38 & 0.227 \\
\hline & & & \\
\hline
\end{tabular}

Methadone

\section{Fluid deliveries}

In general, exposure to a range of methadone concentrations $(0.001-0.3 \mathrm{mg} / \mathrm{ml})$ had no effect on methadone or water fluid deliveries. There were no changes in, or differences between, methadone and water fluid deliveries (Fig. 4, top panels). No gender differences (main effects and interactions) were demonstrated with methadone or water fluid deliveries (Table 3).

\section{Intake}

Self-administered methadone intake $(\mathrm{mg} / \mathrm{kg})$ increased as a function of methadone concentration, reaching significance at the $0.1-\mathrm{mg} / \mathrm{ml}$ concentration (Fig. 4, bottom panels). Maximal methadone intake was demonstrated at the highest tested concentration $(0.3 \mathrm{mg} / \mathrm{ml})$, in which NIAAA and MICH monkeys consumed approximately $4 \mathrm{mg} / \mathrm{kg}$ and $1 \mathrm{mg} / \mathrm{kg}$, respectively. There were no gender differences in methadone intake. 
Fig. 5 Top panels: pentobarbital (filled symbols) and water (hollow symbols) fluid deliveries (per session) as a function of pentobarbital concentration ( $\mathrm{mg} / \mathrm{ml})$. Unconnected symbols indicate the fluid deliveries obtained in sessions in which water was concurrently available from both spouts. Bottom panels: pentobarbital intake $(\mathrm{mg} / \mathrm{kg}$ per session) as a function of pentobarbital concentration $(\mathrm{mg} / \mathrm{ml})$. Left and right panels depict NIAAA and MICH monkeys, respectively. For all panels, each point represents the mean of six sessions, and error bars denote the 95\% confidence interval associated with the mean

Table 4 Oral pentobarbital self-administration in rhesus monkeys

*Significant $F$ value $(P<0.05)$ for comparison
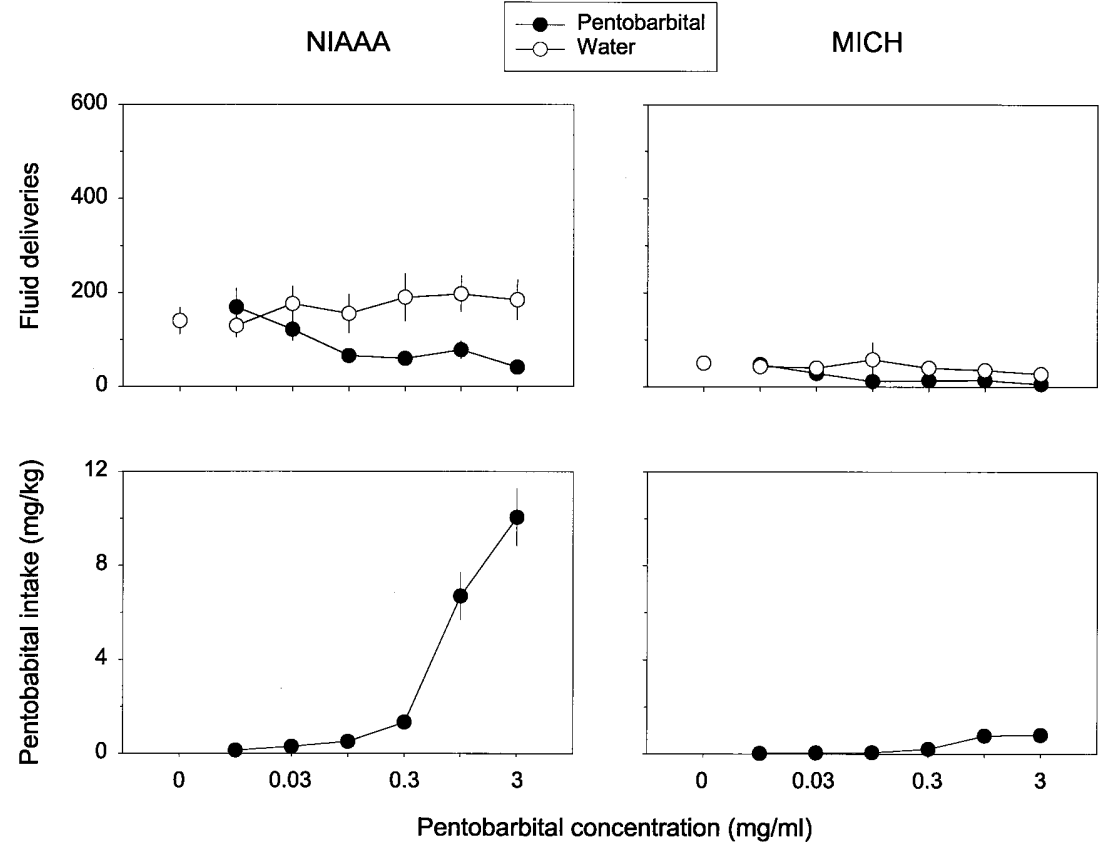

\begin{tabular}{llcl}
\hline Factor & df & $F$ value & $P$ value \\
\hline Fluid deliveries & & & \\
Group & 1,20 & 7.31 & 0.140 \\
Gender & 1,20 & 0.002 & 0.967 \\
Drug & 1,20 & 24.89 & $0.001^{*}$ \\
Concentration & 6,120 & 4.62 & $0.001^{*}$ \\
Group $\times$ Gender & 1,20 & 0.35 & 0.561 \\
Group $\times$ Drug & 1,20 & 8.49 & $0.009^{*}$ \\
Group $\times$ Concentration & 6,120 & 1.30 & 0.262 \\
Gender $\times$ Drug & 1,20 & 6.73 & $0.017^{*}$ \\
Gender $\times$ Concentration & 6,120 & 0.32 & 0.926 \\
Drug $\times$ Concentration & 6,120 & 11.05 & $0.001^{*}$ \\
Group $\times$ Gender $\times$ Drug & 1,20 & 0.99 & 0.331 \\
Group $\times$ Gender $\times$ Concentration & 6,120 & 1.87 & 0.092 \\
Group $\times$ Drug $\times$ Concentration & 6,120 & 5.32 & $0.001^{*}$ \\
Gender $\times$ Drug $\times$ Concentration & 6,120 & 1.50 & 0.184 \\
Group $\times$ Gender $\times$ Drug $\times$ Concentration & 6,120 & 0.99 & 0.437 \\
Intake & & & \\
Group & & & \\
Gender & 1,20 & 3.49 & 0.077 \\
Concentration & 1,20 & 0.37 & 0.550 \\
Group $\times$ Gender & 6,120 & 4.95 & $0.001^{*}$ \\
Group $\times$ Concentration & 1,20 & 0.62 & 0.440 \\
Gender $\times$ Concentration & 6,120 & 3.44 & $0.004^{*}$ \\
Group $\times$ Gender $\times$ Concentration & 6,120 & 0.40 & 0.877 \\
\hline & 6,120 & 0.66 & 0.681 \\
\hline
\end{tabular}

\section{Pentobarbital}

\section{Fluid deliveries}

NIAAA and MICH monkeys obtained more water than pentobarbital $(0.03-3 \mathrm{mg} / \mathrm{ml})$ fluid deliveries, reaching significance at the $0.1-\mathrm{mg} / \mathrm{ml}$ concentration. This effect was reflected in the reduction of pentobarbital fluid deliveries obtained (Fig. 5, top panels). No gender differences (main effects and interactions) were demonstrated with pentobarbital or water fluid deliveries (Table 4).

\section{Intake}

Self-administered pentobarbital intake $(\mathrm{mg} / \mathrm{kg})$ increased as a function of pentobarbital concentration, reaching significance at $1 \mathrm{mg} / \mathrm{ml}$ (Fig. 5, bottom panels). Maximal pentobarbital intake was demonstrated at the highest tested concentration ( $3 \mathrm{mg} / \mathrm{ml})$, in which NIAAA and $\mathrm{MICH}$ monkeys consumed approximately $10 \mathrm{mg} / \mathrm{kg}$ and $0.75 \mathrm{mg} / \mathrm{kg}$, respectively. There were no gender differences in pentobarbital intake. 
Fig. 6 Top panels: quinine (filled symbols) and water (hollow symbols) fluid deliveries (per session) as a function of quinine concentration $(\mathrm{mg} / \mathrm{ml})$. Unconnected symbols indicate the fluid deliveries obtained in sessions in which water was concurrently available from both spouts. Bottom panels: quinine intake $(\mathrm{mg} / \mathrm{kg}$ per session) as a function of quinine concentration $(\mathrm{mg} / \mathrm{ml})$. Left and right panels depict NIAA and MICH monkeys, respectively. For all panels, each point represents the mean of six sessions, and error bars denote the 95\% confidence interval associated with the mean
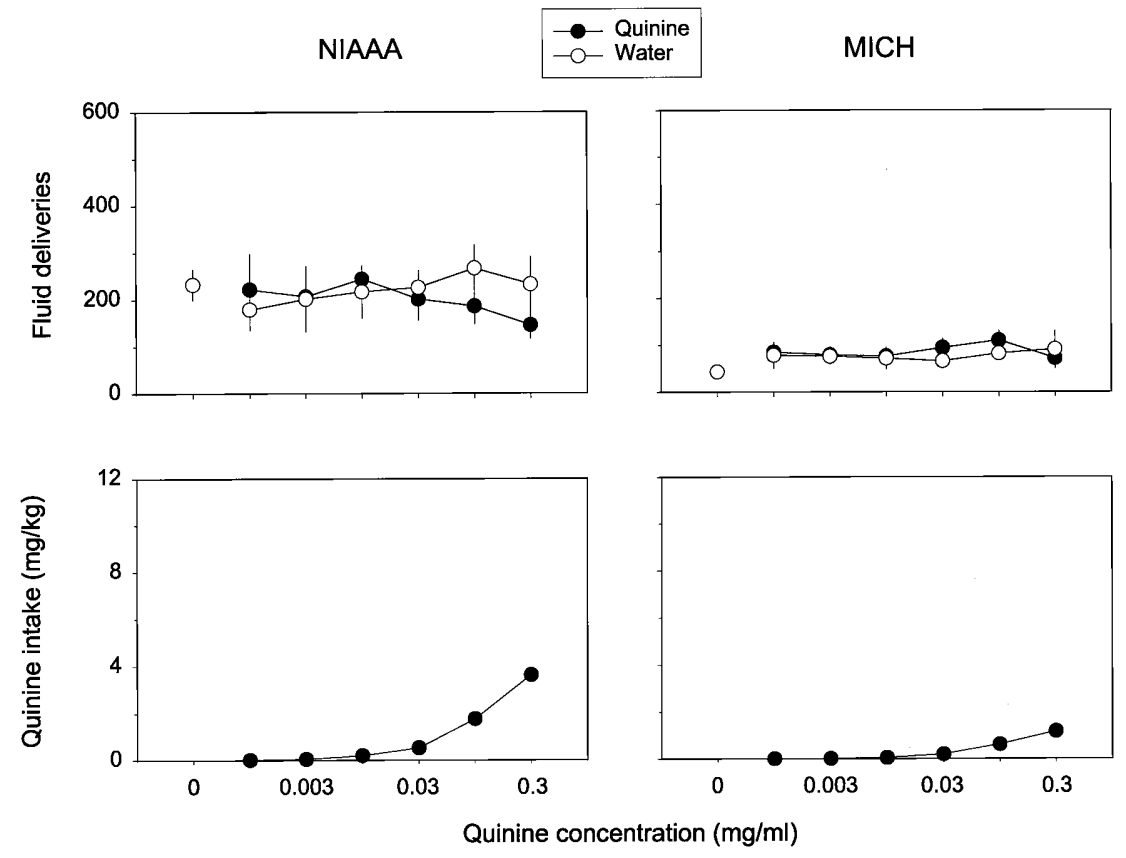

Table 5 Oral quinine self-administration in rhesus monkeys

* Significant $F$ value $(P<0.05)$ for comparison

\begin{tabular}{llll}
\hline Factor & df & $F$ value & $P$ value \\
\hline Fluid deliveries & & & \\
Group & 1,20 & 8.15 & $0.010^{*}$ \\
Gender & 1,20 & 0.64 & 0.803 \\
Drug & 1,20 & 0.01 & 0.930 \\
Concentration & 6,120 & 0.49 & 0.817 \\
Group $\times$ Gender & 1,20 & 1.13 & 0.301 \\
Group $\times$ Drug & 1,20 & 0.05 & 0.825 \\
Group $\times$ Concentration & 6,120 & 2.49 & 0.263 \\
Gender $\times$ Drug & 1,20 & 0.73 & 0.402 \\
Gender $\times$ Concentration & 6,120 & 0.34 & 0.916 \\
Drug $\times$ Concentration & 6,120 & 1.11 & 0.359 \\
Group $\times$ Gender $\times$ Drug & 1,20 & 1.33 & 0.263 \\
Group $\times$ Gender $\times$ Concentration & 6,120 & 0.80 & 0.573 \\
Group $\times$ Drug $\times$ Concentration & 6,120 & 0.55 & 0.767 \\
Gender $\times$ Drug $\times$ Concentration & 6,120 & 0.41 & 0.873 \\
Group $\times$ Gender $\times$ Drug $\times$ Concentration & 6,120 & 0.65 & 0.690 \\
Intake & & & \\
Group & 1,20 & 12.18 & $0.002^{*}$ \\
Gender & 1,20 & 1.35 & 0.259 \\
Concentration & 6,120 & 39.59 & $0.001 *$ \\
Group $\times$ Gender & 1,20 & 0.09 & 0.767 \\
Group $\times$ Concentration & 6,120 & 9.38 & $0.001 *$ \\
Gender $\times$ Concentration & 6,120 & 0.76 & 0.602 \\
Group $\times$ Gender $\times$ Concentration & 6,120 & 0.04 & 0.999 \\
\hline
\end{tabular}

Quinine

\section{Fluid deliveries}

Exposure to a range of quinine concentrations $(0.001-0.3 \mathrm{mg} / \mathrm{ml})$ had no effect on quinine or water fluid deliveries. There were no changes in, or differences between, methadone and water fluid deliveries (Fig. 6, top panels). No gender differences (main effects and interactions) were demonstrated with quinine or water fluid deliveries (Table 5).

\section{Intake}

Self-administered quinine intake $(\mathrm{mg} / \mathrm{kg}$ ) increased as a function of quinine concentration, reaching significance at $0.1 \mathrm{mg} / \mathrm{ml}$ (Fig. 6, bottom panels). Maximal quinine intake was demonstrated at the highest tested concentration $(0.3 \mathrm{mg} / \mathrm{ml})$, in which NIAAA and $\mathrm{MICH}$ monkeys consumed approximately $3.75 \mathrm{mg} / \mathrm{kg}$ and $1 \mathrm{mg} / \mathrm{kg}$, respectively. There were no gender differences in quinine intake. 


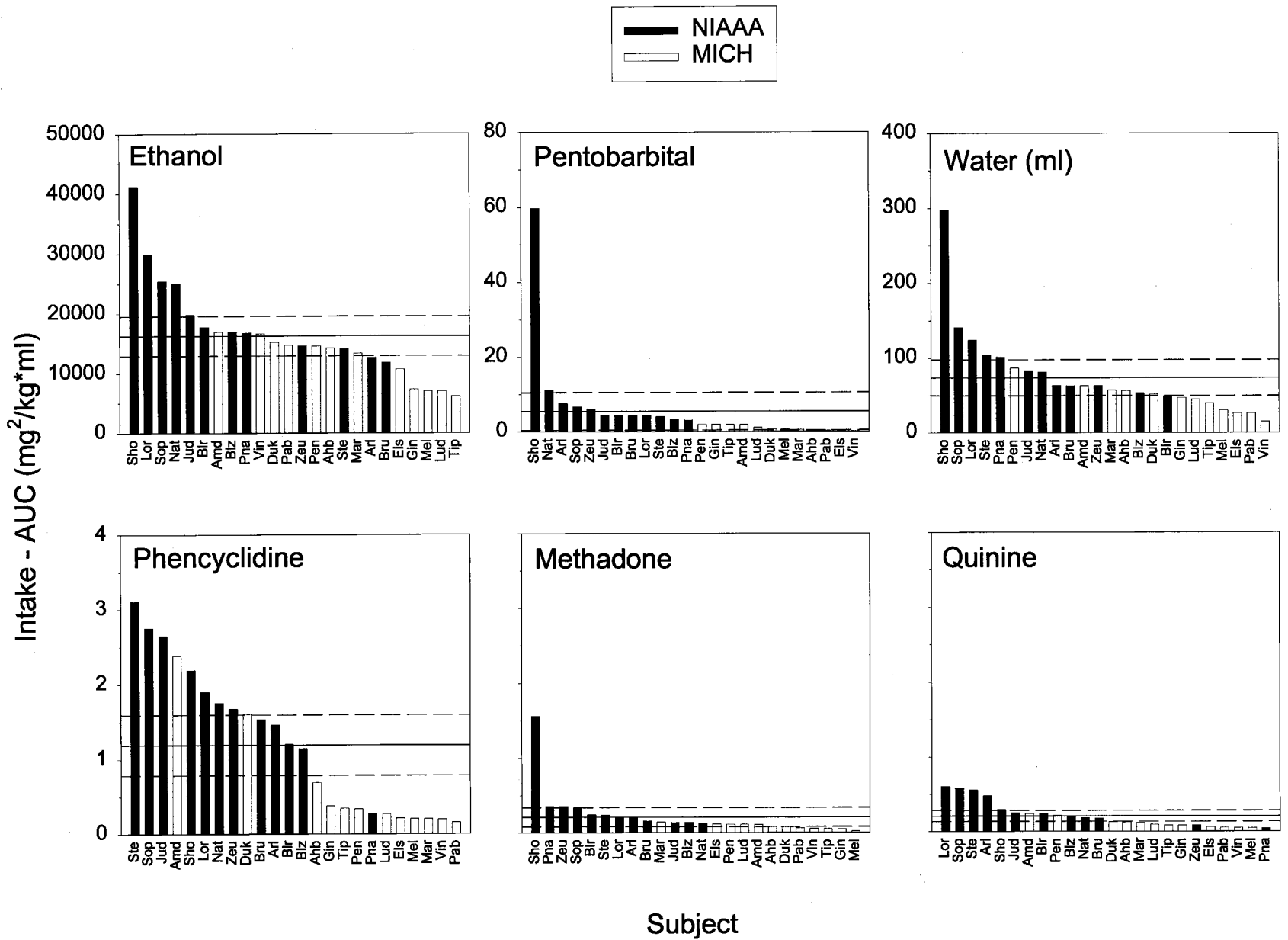

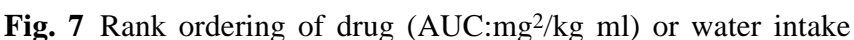
(ml) for each monkey. Dashed lines indicate the 95\% confidence intervals associated with the group mean. Filled bars indicate NIAAA, and hollow bars indicate MICH monkeys. See Methods for calculation of AUC

\section{Correlations among self-administered drugs}

In an effort to determine whether self-administration of one drug was correlated with another, preference and intake data for each subject and drug were transformed into AUC values, or preference or aversion values (drug/water fluid deliveries or water/drug fluid deliveries, respectively) were calculated at the drug concentration that produced the largest preference or aversion, respectively. Subsequently, each subject was rank-ordered based on these preferences and intakes, and Spearman correlation coefficients were derived across drugs. Consistent with the mean intake data described above, NIAAA monkeys had higher AUC intakes (were ranked higher) than MICH monkeys (Fig. 7).

\section{Drug preference}

Overall, rank-order preference for one drug was not correlated with the preference for another (Table 6). Calcu- lations based on AUC revealed that phencyclidine preference was correlated with quinine preference $\left[r_{\mathrm{s}}(24)=0.55, P<0.05\right]$. Similarly, correlations based on the concentrations in which the largest mean preference or aversions were demonstrated were also unremarkable $\left[-0.3<r_{\mathrm{s}}(24)<0.3\right.$; data not shown]. The only exceptions were that ethanol preferences were correlated with ethanol aversions $\left[r_{\mathrm{s}}(24)=-0.48, \quad P<0.05\right]$; phencyclidine preferences were correlated with quinine preferences $\left[r_{\mathrm{s}}(24)=0.56, P<0.05\right]$; quinine preferences were correlated with quinine $\left[r_{\mathrm{s}}(24)=-0.57, P<0.05\right]$ and pentobarbital $\left[r_{\mathrm{s}}(24)=0.46, P<0.05\right]$ aversions.

\section{Drug intake}

Initial correlation matrixes revealed significant correlations among all of the compounds tested [all drugs: $\left.r_{\mathrm{s}}(24)>0.5, P<0.05\right]$. Yet drug intakes (in $\mathrm{mg} / \mathrm{kg}$ ) were also related to the baseline consumption of water (in $\mathrm{ml}$ ) in water-water choice conditions $\left[r_{\mathrm{s}}(24)>0.6, P<0.05\right]$. After partialling out the effects of baseline fluid consumption, phencyclidine intake was found to correlate with pentobarbital $\left[r_{\mathrm{s}}(24)=0.42, P<0.05\right]$ and quinine intake $\left[r_{s}(24)=0.58, P<0.05\right]$. Additional correlations within the NIAAA and MICH groupings revealed that phencyclidine intake was correlated with quinine intake 
Table 6 Oral self-administration of ethanol, phencyclidine, methadone, pentobarbital and quinine in rhesus monkeys: spearman correlations between drugs
Values derived from area under the curve calculations (see Methods). *Significant $(P<0.05)$ correlation coefficients

\begin{tabular}{|c|c|c|c|c|c|}
\hline & Ethanol & Phencyclidine & Methadone & Pentobarbital & Quinine \\
\hline \multicolumn{6}{|l|}{ A. Preference } \\
\hline Phencyclidine & 0.06 & & & & \\
\hline Methadone & -0.03 & 0.36 & & & \\
\hline Pentobarbital & -0.11 & 0.15 & 0.38 & & \\
\hline Quinine & -0.08 & $0.55^{*}$ & -0.02 & -0.26 & \\
\hline \multicolumn{6}{|l|}{ B. Intake } \\
\hline Phencyclidine & $0.55 *$ & & & & \\
\hline Methadone & $0.52 *$ & $0.52 *$ & & & \\
\hline Pentobarbital & $0.49 *$ & $0.74 *$ & $0.71 *$ & & \\
\hline Quinine & $0.51 *$ & $0.80 *$ & $0.49 *$ & $0.64 *$ & \\
\hline Water (ml) & $0.57 *$ & $0.74 *$ & $0.74 *$ & $0.72 *$ & $0.72 *$ \\
\hline \multicolumn{6}{|c|}{ C. Intake (water partialled out) } \\
\hline Phencyclidine & 0.23 & & & & \\
\hline Methadone & 0.17 & -0.06 & & & \\
\hline Pentobarbital & 0.13 & $0.42 *$ & 0.35 & & \\
\hline Quinine & 0.19 & $0.57 *$ & -0.10 & 0.24 & \\
\hline
\end{tabular}

[NIAAA: $\quad r_{\mathrm{s}}(12)=0.64, \quad P<0.05 ; \quad \mathrm{MICH}: \quad r_{\mathrm{s}}(12)=0.77$, $P<0.05]$

\section{Discussion}

The present investigation characterized the oral self-administration propensities for various drugs using a large group of monkeys with varied drug preferences and intakes. Implicit in this characterization was the evaluation of the relationship between orally available solutions with reinforcing effects in a self-administration paradigm, e.g., do the preferences for, or intakes of, one drug predict the preferences for, or intakes of, another? In the current study, preferences for ethanol were robustly and reliably demonstrated, whereas preferences for drugs from other classes were not. After rank ordering each monkey based on its drug preference or intake, moderate correlations between drug intakes were demonstrated. This result suggests that monkeys that consume large amounts of one drug continue to consume large amounts of another drug. Stated differently, the intake of one drug predicted the intake of another. However, drug intakes were also correlated with baseline intakes of water in water-water choice conditions. When these baseline intakes were partialled out, intake of one drug was not correlated with intake of another. This result argues against the above proposal and suggests that fluid intakes were correlated, irrespective of the presence or absence of drug in the solution.

The failure to demonstrate appreciable correlations between preferences and intakes across drugs was somewhat surprising, and the relevance of the present investigation involving exposure to drugs from multiple drug classes to human populations should not be overlooked. In clinical populations, dual alcohol and drug problem diagnoses are common (Weisner 1992; Martin et al. 1996), and the occurrence of a "pure" substance user an individual who uses, for example, ethanol or heroin exclusively - is rare (Darke and Hall 1995; Miller et al. 1997). In fact, comorbidity among multiple substance abuse disorders is universal in some populations (Busto et al. 1996). In addition, the basis of many "gateway" theories is that use and initiation of one drug, commonly nicotine or alcohol, predicts future and other drug use (Yu and Williford 1992; Torabi et al. 1993). In the current investigation, there were no correlations between the individual preferences and intakes of each drug. These results suggest that simple exposure to a drug is not sufficient to establish reinforcing effects, nor is it a controlling factor in an organism's propensity to prefer or consume other reinforcing drugs.

In contrast, reports of correlations between preferences and intakes across various self-administered solutions including saccharin and ethanol (Bell et al. 1994; Gahtan et al. 1996), saccharin and morphine (Gosnell et al. 1995) and cocaine and ethanol (Gahtan et al. 1996) have been accumulating in rats, leading to the hypothesis that drug-seeking behavior may have common biological determinants (George 1993). Yet there is also evidence for an independence in preferences or intakes across various drugs in self-administration research. There were no relationships demonstrated between the preferences of orally available etonitazene, cocaine and ethanol in rats with a preference for etonitazene over water (Carlson and Perez 1997). Likewise, no relationship was demonstrated between saccharin and cocaine in rats with a preference for saccharin (Gahtan et al. 1996; Gosnell et al. 1998). Interestingly, non-human primate research has not thoroughly or explicitly tested the relationship between the preferences for, and intakes of, one drug and another. In rhesus monkeys with an extensive ethanol oral selfadministration history, preferences and consumption of ethanol were not correlated with subsequent preferences and consumption of oral cocaine or midazolam (Meisch et al. 1990; Stewart et al. 1994). These latter investigations involving rhesus monkeys were consistent with the results from the present investigation in that preferences 
and consumption of one drug failed to predict the preferences and consumption of another. As these previous primate studies were not explicitly designed to compare the self-administration of one drug with another, some caution must be noted as to their relevance, but together with the equivocal self-administration evidence in rats, the need for future research into this topic is highlighted.

One concern about the present investigation is the notable lack of preferences for any of the drug solutions other than ethanol. It must be pointed out that a preference for water over a drug, or no preference at all, does not necessarily indicate that the drug does not serve as a positive reinforcer, but simply that water was preferred over drug. If the drug was not serving as a positive reinforcer, behavior directed toward the drug would not be maintained. Nonetheless, future research should consider alternatives to increase the preferences for the drug choices, including increasing the schedule maintaining the behavior, schedule-induced polydipsia, fading or food restriction strategies, to address the relationship between drug preferences and reinforcing effects.

In addition, the failure to demonstrate preferences for, or correlations among the intakes of, the various drugs may simply be the result of a lack of pharmacological or behavioral activity at the doses consumed. This is unlikely as behavioral effects, including ataxia and respiratory suppression, were noted in individual subjects when exposed to higher drug concentrations. Moreover, the mean and range of intakes at the higher concentrations from the present investigation were consistent with previous oral self-administration research involving phencyclidine (present study: ca. $7 \mathrm{mg} / \mathrm{kg}$; Carroll 1982: ca. $10 \mathrm{mg} / \mathrm{kg}$ ), methadone (present study: $4 \mathrm{mg} / \mathrm{kg}$; Stewart et al. 1996: $5 \mathrm{mg} / \mathrm{kg}$ ), with the exception of pentobarbital (present study: ca. $10 \mathrm{mg} / \mathrm{kg}$; Meisch et al. 1981; ca. $33 \mathrm{mg} / \mathrm{kg}$ ). Interestingly, these latter studies demonstrated drug preferences over water and utilized different experimental parameters, including controlled food intake and schedules controlling behavior.

Drug use patterns among clinical populations suggests that multiple-drug use, whether simultaneous or sequential, is the norm, whereas drug self-administration patterns among rodent and non-human primate populations have not been thoroughly explored. In the present investigation, rhesus monkeys were exposed to a variety of drugs with previously established reinforcing effects. These monkeys demonstrated the reinforcing effects of ethanol, and at the higher concentrations, consumed pharmacologically relevant doses of each compound tested. Nonetheless, and irrespective of an individual monkey's propensity to consume a drug, the self-administration of one drug failed to predict self-administration of the other drugs.

Acknowledgements Supported by USPHS Grants DA00254 and DA05773, and by NIAAA. Animals used in these studies were maintained in accordance with the University of Michigan Committee on Animal Care and Guidelines of the Committee on the Care and Use of Laboratory Animal Resources, National Health Council (Department of Health, Education and Welfare, ISBN 0-309-05377-3, revised 1996).

\section{References}

Aspen J, Gatch MB, Woods JH (1999) Training and characterization of a quinine taste discrimination in rhesus monkeys. Psychopharmacology 141:251-257

Bell SM, Gosnell BA, Krahn DD, Meisch RA (1994) Ethanol reinforcement and its relationship to saccharin preference in $\mathrm{Wi}$ star rats. Alcohol 11:141-145

Busto UE, Romach MK, Sellers EM (1996) Multiple drug use and psychiatric comorbidity in patients admitted to the hospital with severe benzodiazepine dependence. J Clin Psychopharmacol 16:51-57

Cadoret RJ (1993) Genetic and environmental factors in initiation of drug use and the transition to abuse. In: Glantz M, Pickens $\mathrm{R}$ (eds) Vulnerability to drug abuse. American Psychological Association, Washington DC, pp 99-114

Carlson KR, Perez L (1997) Ethanol and cocaine intake by rats selectively bred for oral opioid acceptance. Pharmacol Biochem Behav 57:309-313

Carroll ME (1982) Oral self-administration of phencyclidine analogs by rhesus monkeys: conditioned taste and visual reinforcers. Psychopharmacology 78:116-120

Darke S, Hall W (1995) Levels and correlates of polydrug use among heroin users and regular amphetamine users. Drug Alcohol Depend 39:231-235

Gahtan E, Labounty LP, Wyvell C, Carroll ME (1996) The relationship among saccharin consumption, oral ethanol and i.v. cocaine self-administration. Pharmacol Biochem Behav 53: 919-925

George FR (1993) Genetic models in the study of alcoholism and substance abuse mechanisms. Prog Neuropsychopharmacol Biol Psychiatry 17:345-361

Gosnell BA, Lane KE, Bell SM, Krahn DD (1995) Intravenous morphine self-administration by rats with low versus high saccharin preferences. Psychopharmacology 117:248-252

Gosnell BA, Krahn DD, Yracheta JM, Harasha BJ (1998) The relationship between intravenous cocaine self-administration and avidity for saccharin. Pharmacol Biochem Behav 60: 229-236

Henningfield JE, Meisch RA (1978) Ethanol drinking by rhesus monkeys as a function of concentration. Psychopharmacology $57: 133-136$

Higley JD, Hasert MF, Suomi SJ, Linnoila M (1991) Nonhuman primate model of alcohol abuse: effects of early experience, personality, and stress on alcohol consumption. Proc Natl Acad Sci USA 88:7261-7265

Higley JD, Suomi SJ, Linnoila M (1996) A nonhuman primate model of type II excessive alcohol consumption? Part 1. Low cerebrospinal fluid 5-hydroxyindoleacetic acid concentrations and diminished social competence correlate with excessive alcohol consumption. Alcohol Clin Exp Res 20:629-642

Kachigan SK (1986) Statistical analysis. Radius Press, New York

Martin CS, Clifford PR, Maisto SA, Earleywine M, Kirisci L, Longabaugh R (1996) Polydrug use in an inpatient treatment sample of problem drinkers. Alcohol Clin Exp Res 20:413-417

Meisch RA, Kliner DJ, Henningfield JE (1981) Pentobarbital drinking by rhesus monkeys: establishment and maintenance of pentobarbital-reinforced behavior. J Pharmacol Exp Ther 217:114-120

Meisch RA, George FR, Lemaire GA (1990) Orally delivered cocaine as a reinforcer for rhesus monkeys. Pharmacol Biochem Behav 35:245-249

Merikangas KR, Rounsaville BJ, Prusoff BA (1993) Familial factors in vulnerability to substance abuse. In: Glantz M, Pickens $\mathrm{R}$ (eds) Vulnerability to drug abuse. American Psychological Association, Washington DC, pp 75-98

Miller NS, Guttman JC, Chawla S (1997) Integration of generalized vulnerability to drug and alcohol addiction. J Addict Dis 16:7-22

Newcomb MD (1993) Understanding the multidimensional nature of drug use and abuse: the role of consumption, risk factors, and protective factors. In: Glantz M, Pickens R (eds) Vulnera- 
bility to drug abuse. American Psychological Association, Washington DC, pp 255-298

Stewart RB, Lemaire GA, Roache JD, Meisch RA (1994) Establishing benzodiazepines as oral reinforcers: midazolam and diazepam self-administration in rhesus monkeys. J Pharmacol Exp Ther 271:200-211

Stewart RB, Grabowski J, Wang NS, Meisch RA (1996) Orally delivered methadone as a reinforcer in rhesus monkeys. Psychopharmacology 123:111-118

Tallarida RJ, Murray RB (1986) Manual of pharmacologic calculations with computer programs, 2nd edn. Springer, Berlin Heidelberg, New York

Tarter RE, Mezzich AC (1993) Ontogeny of substance abuse: perspectives and findings. In: Glantz M, Pickens R (eds) Vulnerability to drug abuse. American Psychological Association, Washington DC, pp 149-178

Torabi MR, Bailey WJ, Majd-Jabbari M (1993) Cigarette smoking as a predictor of alcohol and other drug use by children and adolescents: evidence of the "gateway drug effect". J School Health 63:302-306

Vivian JA, Higley JD, Linnoila M, Woods JH (1999a) Ethanol oral self-administration in rhesus monkeys: behavioral and neurochemical correlates. Alcohol Clin Exp Res (in press)

Vivian JA, Sumpter TL, Woods JH (1999b) Further characterization of the quinine taste discriminative stimulus in rhesus monkeys: effects of inter-trial interval, number and distribution of test stimuli. Behav Pharmacol 10:313-318

Weisner C (1992) A comparison of alcohol and drug treatment clients: are they from the same population? Am J Drug Alcohol Abuse 18:429-444

Yu J, Williford WR (1992) The age of alcohol onset and alcohol, cigarette, and marijuana use patterns: an analysis of drug use progression of young adults in New York State. Int $\mathbf{J}$ Addict $27: 1313-1323$ 